INPLASY

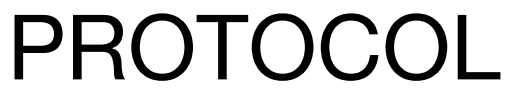

To cite: Tang et al. The effects of Huaier granule in adjuvant therapy after curative resection of colorectal cancer: A protocol for systematic review and meta-analysis. Inplasy protocol 202130041. doi: 10.37766/inplasy2021.3.0041

Received: 13 March 2021

Published: 13 March 2021

Corresponding author:

Shengnan Tang

450606486@qq.com

Author Affiliation:

Gansu University of Chinese Medicine

Support: Yes.

Review Stage at time of this submission: The review has not yet started.

Conflicts of interest:

None declared.

\section{The effects of Huaier granule in adjuvant therapy after curative resection of colorectal cancer: A protocol for systematic review and meta-analysis}

Tang, SN1; Teng, R²; Zhao, DS3; Nie, GY4; Zhang, WJ5.

Review question / Objective: The aim of this study is to assess the comparative efficacy and safety of Huaier granule in adjuvant therapy after curative resection of colorrectal cancer through systematic review and meta-analysis.

Condition being studied: Colorectal cancer (CRC) is the third most commonly diagnosed cancer worldwide. Surgical resection is the primary treatment modality for stage I-III colorectal cancer, with systemic chemotherapy or radiotherapy. Huaier granule have been proven to be effective against a variety of cancers. However, its anti-CRC effect in the clinic remains controversial and requires additional research.

INPLASY registration number: This protocol was registered with the International Platform of Registered Systematic Review and Meta-Analysis Protocols (INPLASY) on 13 March 2021 and was last updated on 13 March 2021 (registration number INPLASY202130041).

\section{INTRODUCTION}

Review question / Objective: The aim of this study is to assess the comparative efficacy and safety of Huaier granule in adjuvant therapy after curative resection of colorrectal cancer through systematic review and meta-analysis.
Rationale: Systematic review and metaanalysis.

Condition being studied: Colorectal cancer (CRC) is the third most commonly diagnosed cancer worldwide. Surgical resection is the primary treatment modality for stage I-III colorectal cancer, with systemic chemotherapy or radiotherapy. 
Huaier granule have been proven to be effective against a variety of cancers. However, its anti-CRC effect in the clinic remains controversial and requires additional research.

\section{METHODS}

Search strategy: PubMed, Cochrane Library, Embase, Web of Science, Chinese National Knowledge Infrastructure, WanFang database, China Biology Medicine Database and Chongqing VIP Chinese Scientific Journals Full-text Database.

Participant or population: The target population was patients with primary CRC, using the definitions of the first episodes selected by the original study authors. These patients with CRC treated surgically with curative intent. Those who had local excision of tumors, distant metastasis, or other colorectal pathology, e.g., inflammatory bowel disease, will be excluded. The patient must be an adult (age $\geq 18$ years). There will no restriction on gender and race.

Intervention: Observation group patients must be treated with Huaier granule, whether given alone or in combination with chemotherapy and/or radiation therapy, against chemotherapy and/or radiation therapy alone, a placebo, or no treatment. Control group patients cannot receive Huaier granule treatment, other interventions must be completely consistent with the observation group. In different studies, the dosage and course of treatment of Huaier granules used by patients can be different.

Comparator: Observation group patients must be treated with Huaier granule, whether given alone or in combination with chemotherapy and/or radiation therapy, against chemotherapy and/or radiation therapy alone, a placebo, or no treatment. Control group patients cannot receive Huaier granule treatment, other interventions must be completely consistent with the observation group. In different studies, the dosage and course of treatment of Huaier granules used by patients can be different.

Study designs to be included: We plan to included randomized controlled trials (RCTs) and controlled clinical trials (CCTs) that compared the adjuvant therapeutic effect of Huaier granule in patients after curative resection of CRC. Qualitative studies, observational studies, and case reports will be excluded. If the data cannot be obtained by contacting the author, the studies reported in abstract form that without presenting the outcome will be excluded.

Eligibility criteria: See the items (Participant or population; Intervention; Comparator; and Study designs to be included.

Information sources: The databases are as follows: PubMed, Cochrane Library, Embase, Web of Science, Chinese National Knowledge Infrastructure, WanFang database, China Biology Medicine Database and Chongqing VIP Chinese Scientific Journals Full-text Database. If the data is incomplete, the authors of the studies will be contacted through email or telephone. In addition, reference lists in the relevant publications, such as reviews conducted on of Huaier granule for CRC, will also be hand checked for search additional eligible trials.

Main outcome(s): The treatment response rate;Overall survival at 0.5 -year $(0.5-0 S)$, one-year (1-OS), three years (3-OS), and five years (5-OS); Karnofsky Performance Status improvement rate.

Additional outcome(s): The local tumor treatment response: complete response, partial response, progressive disease, and stable disease; Disease progression at one year confirmed radiologically or biopsy proven; if data are available, will plus three years or five years; Disease recurrence at one year confirmed radiologically or biopsy proven; if data are available, will plus three years or five years; Quality of life evaluated using any validated assessment tool; Immune function: the levels of CD3+, 
CD4+, CD8+, CD4+/CD8+, NK cells, IL-2, IgA, IgG and IgM;Adverse effect rates: decreases in leukocytes and platelets, nausea and vomiting, debilitation, diarrhea and stomachache.

Data management: We will extract and summarize details of the eligible studies using a pre-designed and calibrated standardized data extraction sheet. The calibration process is as follows: using a random sample of five of the included studies, the data extraction form will be pilot-tested, and revised as necessary. We will extract data on the following items: name of the first author, year of publication, study location, study design, study setting, participants, sample size, follow-up period, outcomes of interest, risk and prognostic factors, missing data, summary statistics, outcomes and interpretation. Two reviewers will extract data independently and will resolve disagreements by discussion, drawing on a third review author when required. If the data is incomplete, the authors of the studies will be contacted through email or telephone. Data will be extracted by one author and double-checked by a second author.

Quality assessment / Risk of bias analysis: The Cochrane Risk of Bias (RoB) Tool for RCTs will be used to evaluate the risk of bias of each included RCT and quasirandomized trial. And the quality of CCTs will be assessed by the Newcastle-Ottawa Scale (NOS) that used as suggested by the Cochrane Non-Randomized Studies Methods Working Group. The RoB tool has seven domains and each aspect is judged as three levels: high risk of bias, unclear risk of bias, and low risk of bias. All domains will be considered, including sequence generation and allocation sequence concealment (selection bias), blinding of participants and personnel (performance bias), blinding of outcome assessment (detection bias), incomplete outcome data (attrition bias), selective reporting (reporting bias), and other biases considered relevant to the review topic. NOS utilized the following questions: (1) Is the case definition adequate? (2)
Representativeness of the cases? (3) Selection of Controls? (4) Definition of Controls? (5) Comparability of cases and controls (0-2)? (6) Ascertainment of exposure (0-2)? (7) Nonresponse rate? We will exclude the studied if the total scores less than five. Two reviewers will independently assess the quality of included studies. Any disagreements will be resolved by consensus or with a third reviewer.

Strategy of data synthesis: We will use a summary table to descriptive the primary study characteristics, such as sample size, sample mean age, percentage of female participants, and study publication year. The software Stata (version 14.0) will be used to a random-effects meta-analysis. About the study effect size, we will calculate the odds ratios for binary outcomes and standardized mean differences for continuous outcomes, with $95 \%$ confidence interval.

Subgroup analysis: If heterogeneity is high, we will perform a subgroup analysis. And if cannot perform subgroup analysis, We will performer a descriptive analysis.

Sensitivity analysis: If heterogeneity is high, we will perform a subgroup analysis. And if cannot perform subgroup analysis, We will performer a descriptive analysis.

\section{Language: None.}

Country(ies) involved: China.

Other relevant information: None.

Keywords: Huaier granule, adjuvant therapy, colorectal cancer, systematic review, meta-analysis.

Dissemination plans: If the data is incomplete, the authors of the studies will be contacted through email or telephone.

Contributions of each author:

Author 1 - Shengnan Tang - Data curation; Project administration; Writing-original draft.

Email: 450606486@qq.com 
Author 2 - Ran Teng - Formal analysis;

Resources; Writing-review \& editing.

Email: 136369968@qq.com

Author 3 - Dangsheng Zhao - Funding acquisition.

Email: zhds69@126.com

Author 4 - Guiyuan Nie - Software.

Email: 450606486@qq.com

Author 5 - WenJun Zhang - Project administration; Resources; Supervision;

Validation Writing-review \& editing.

Email: 1272025508@qq.com 